\title{
Florent Garnier
}

\section{La résolution des conflits commerciaux d'après les conférences régionales des juges consulaires: L'exemple de la 1 ère conférence régionale de Riom en $1899^{*}$}

Le décret du 15 février 2008 modifiant le siège et le ressort des tribunaux de commerce prévoyait en son article 1er la suppression, à compter du $1^{\mathrm{er}}$ janvier 2009, de plusieurs juridictions consulaires. Dans le ressort de la Cour d'appel de Riom, trois tribunaux de commerce ont disparu (Moulins, Riom et Thiers). Pour l'Auvergne, l'histoire de ces juridictions consulaires et celle droit commercial sont en général assez mal connues ${ }^{1}$. Parmi les sources conservées dans les différends dépôts départementaux et à la Bibliothèque Municipale Inter-Universitaire, le Compte rendu de la Conférence régionale des juges consulaires du ressort de la cour d'appel de Riom en $1899^{2}$ présente quelque intérêt pour répondre à l'invitation des organisateurs du colloque de Roscoff quant à la résolution des conflits en matière de commerce.

A l'initiative de l'ancien président de la juridiction consulaire de Lyon, Pierre Vindry, des conférences régionales sont organisées à Lyon à partir de 1898 puis à Rouen, Orléans ou encore Dijon en relation avec la Conférence générale des Tribunaux de Commerce de France instituée un an plus tard. Le 8 mai 1899, les repré-

"Cette recherche s'inscrit dans le cadre d'un Bonus Qualité Recherche de l'Université d'Auvergne, La vie du droit en Auvergne. Histoire et géographie juridique (Moyen Âge - XIX ${ }^{\mathrm{e}}$ siècle), Centre d'Etudes Romanistiques d'Auvergne (E.A. 2147).

1 Laurent Meyniel, Présence des marchands d'Aurillac aux foires de Provins en 1205, dans: Revue de la Haute-Auvergne (1904) 420-421 et (1905) 91-93; Marcel Boudet, Notes pour servir à l'histoire du commerce de la Haute-Auvergne, les marchands d'Aurillac et de SaintFlour aux foires de Champagne et à Montpellier (XIII-XIVe siècle), dans: Revue de la Haute-Auvergne (1913) 326-341 et (1914) 154-165 et 236-245; Georges Esquer, La HauteAuvergne à la fin de l'Ancien Régime. Saint-Flour. La Foraine de Saint-Flour, dans: Revue de la Haute-Auvergne (1905) 381-397 et (1911) 84-94; Jean-Eric Iung, Commerce et marchands en Haute-Auvergne (XVIe-XVIII e siècles) (Enluminures, Bulletin de photothèque et archives cantaliennes 6, Aurillac 1999) 38 et s.; Florent Garnier, Justice et droit commercial en Haute-Auvergne. Bilan et perspectives (Revue de Haute Auvergne-La justice en HauteAuvergne, Aurillac, à paraître).

2 Bibliothèque Municipale et Interuniversitaire Clermont-Ferrand, A 11445; cité comme suit: BMIUCF, A 11445. 
sentants des 13 tribunaux de commerce du ressort de la cour d'appel de Riom ${ }^{3}$ sont réunis au Palais du Tribunal de commerce de Clermont-Ferrand pour instaurer une nouvelle conférence régionale. Parmi les 26 personnes présentes, dont la moitié est issue de la juridiction consulaire clermontoise, on dénombre des présidents de tribunaux de commerce en exercice, d'anciens présidents, le président de la Chambre de commerce de Clermont-Ferrand, ainsi que des juges. Les présidents des tribunaux de Moulins, d'Aurillac et de Billom, bien qu'ils soient absents, adhérent au principe de la Conférence régionale. La séance d'ouverture permet tout d'abord aux membres de la conférence de rappeler l'intérêt d'une réunion annuelle des juges consulaires ${ }^{4}$. S'inspirant de la Conférence d'Orléans, l'assemblée adopte ensuite le règlement de l'organisation d'une telle rencontre 5 . Cette réunion annuelle est ouverte à tous les présidents et magistrats en exercice ainsi que sur leur demande aux anciens présidents et, avec l'accord du président de leur tribunal, aux anciens juges. Elle accueille aussi en tant que collègues invités ayant voix consultative, les magistrats d'un autre ressort judiciaire. Parmi les participants à cette première conférence générale, on note la présence du lyonnais Vindry ainsi que celle de Maurice Chalus, président de la Chambre de Commerce de Clermont-Ferrand. Bien qu'il n'ait pas voix délibérative, ce qu'il regrette publiquement, il intervient à plusieurs reprises. L'un des intérêts de ses interventions tient à ce qu'il fasse partie du Comité de l'Union des banquiers de province qui a pour conseil Charles Lyon-Caen. Chalus rapporte alors l'opinion du commercialiste parisien. Le règlement adopté précise le but d'une telle association. Il s'agit de «créer et d'entretenir des relations agréables et utiles entre tous ses adhérents, d'étudier les mesures les plus propres à assurer l'accomplissement parfait des devoirs des magistrats». Le discours d'ouverture de Morin, président du tribunal de commerce de Clermont-Ferrand, désigné président de la conférence, est plus explicite. Il assigne à l'assemblée le soin d' «étudier en commun les questions intéressant notre juridiction, pour arriver, dans la mesure du possible, à une unification de réglementation et de jurisprudence ${ }^{6}$ et d' «obtenir des réformes». La méthode de travail adoptée reflète cette volonté. Elle repose sur la discussion de rapports d'autres conférences générales, l'intervention de personnalités invitées ou encore de courriers adressés par des présidents de tribunaux de commerce, par exemple de la Haute-Loire, ou de chambres de commerce. Intéressés par la connaissance d'autres pratiques, les juges consulaires enrichissent ainsi leur réflexion et prennent alors position en s'inspirant parfois de celle adoptée par une autre conférence régionale. Par la diffusion du compte rendu à tous les présidents des tribunaux de commerce et à tous magistrats présents et qui ont adhéré à la réunion, ils manifestent ainsi la volonté d'assurer une large publicité à leurs travaux.

3 Riom, Cusset, Montluçon, Moulins, Aurillac, Saint-Flour, Brioude, Le Puy, Ambert, Billom, Clermont-Ferrand, Issoire et Thiers.

4 BMIUCF, A 11445, 13.

5 BMIUCF, A 11445, 17.

6 BMIUCF, A 11445, 13. 
Après le compte rendu de la séance d'ouverture de la Conférence régionale, le document conservé à la Bibliothèque de Clermont-Ferrand se compose de trois parties. La réunion débute par la lecture du rapport de Vindry sur l'étude et la rédaction des jugements7. Elle se poursuit ensuite avec l'examen de quatre questions adressées par la Conférence des Présidents des Tribunaux de commerce. Après un rapport général ${ }^{8}$, les juges consulaires examinent tout d'abord la liquidation judiciaire, puis la proposition de création de tribunaux d'appel spéciaux pour les jugements des tribunaux de commerce, la comptabilité et les honoraires des syndics dans les faillites et liquidations ${ }^{9}$ et enfin l'organisation de la conciliation en matière commerciale. Les membres de l'assemblée se sont ensuite intéressés à quatre thèmes qu'ils ont souhaités porter à la connaissance de la conférence des Présidents des Tribunaux de Commerce de France. Ils débattent ainsi, à partir du rapport présenté par le président du tribunal de commerce de Montluçon ${ }^{10}$, de l'élection des magistrats consulaires, de l'abrogation de l'article 427 du Code de procédure civile, du projet de loi sur les Conseils des Prud'hommes relativement à la compétence de la juridiction consulaire et enfin de la loi du $1^{\text {er }}$ mars 1898 sur les nantissements de fonds de commerce.

A partir de ces thèmes discutés par les juges consulaires, cette première conférence régionale éclaire la naissance d'une organisation de concertation et de proposition qui est préoccupée de l'amélioration du contentieux commercial. Elle permet de saisir à partir de leur activité et de leurs difficultés pratiques, l'état d'esprit des juges consulaires sur leur rôle, les évolutions du droit ou encore les relations entre le contentieux civil et commercial. Cette réflexion se développe alors que des voix s'élèvent pour annoncer la disparition des juridictions commerciales comme «une éventualité probable» 11 . Thaller considère en effet cette juridiction comme un «véritable anachronisme». Symbole de la «justice privée» au sens strict (Albrecht Cordes), elle ne saurait pour le commercialiste français de la fin du $\mathrm{XIX}^{\mathrm{e}}$ siècle survivre car l'«Etat ne saurait se dessaisir du droit supérieur de justice» ${ }^{12}$, correspondant à la «justice publique» (Albrecht Cordes). Cette conférence régionale permet ainsi de saisir différents types de problèmes liés à la résolution des conflits qu'il s'agisse de différents commerciaux, de conflits de compétence avec la juridiction civile ou encore de la «justice privée», au sens large, avec le recours à un mode transactionnel. Intéressante du point de vue procédural et du fond du droit, cette réunion fait aussi état de la perception que les magistrats consulaires ont de leur juridiction. Leur réflexion présente alors un double intérêt.

7 BMIUCF, A 11445, 20-23.

8 BMIUCF, A 11445, 25 et s.

9 BMIUCF, A 11445, 30. Il est décidé que: $1^{\circ}$ ) chaque tribunal est souverain pour la fixation du tarif des faillites; $2^{\circ}$ ) le juge-commissaire doit présenter à la Chambre du Conseil sa taxation avant qu'elle ne devienne définitive; $3^{\circ}$ ) s'il y a une clôture en insuffisance d'actif, les syndics sont tenus de faire payer les frais par le Parquet.

10 BMIUCF, A 11445, 55-57.

11 Eugène-Edmond Thaller, De l'avenir des tribunaux de commerce, dans: Annales de droit commercial (1889) 200-209; cité comme suit: Thaller, De l'avenir.

12 Thaller, De l'avenir (n. 11) 201. 
Elle déborde d'une part le strict cadre des juridictions consulaires du ressort de la cour d'appel de Riom et de leurs problèmes avec la prise en considération de la situation d'autres institutions d'exception. D'autre part, cette réunion, en relation avec la conférence des Présidents des tribunaux de commerce, permet de saisir la place et le rôle des juridictions consulaires dans l'évolution du droit commercial à la fin du XIXe siècle, notamment pour affirmer, réaffirmer ou faire évoluer la délimitation de leur compétence par rapport aux juridictions de droit commun. Les juges consulaires, par leur connaissance de la pratique, affirment ainsi leur volonté d'éclairer l'action du législateur (I). La spécificité du contentieux commercial et de sa procédure les amène également à se déterminer par rapport au juge civil (II).

\section{Les juges consulaires et le législateur}

Enserré dans le Code de commerce de 1807, le droit commercial va évoluer au cours du XIX ${ }^{\mathrm{e}}$ siècle pour tenir compte des évolutions économiques et des besoins de la pratique. Le processus de «décodification» engagé se manifeste de manière diverse sur plus d'un siècle avec le développement de certaines branches du droit, contenues initialement dans le texte de 1807, en dehors du Code soit qu'elles accèdent à l'autonomie soit qu'elles intègrent d'autres champs juridiques (droit des sociétés, faillites et banqueroutes [...]). Le mouvement se manifeste aussi par l'apparition et la présence hors du Code de nouveaux domaines du droit commercial (vente et nantissement du fonds de commerce, bail commercial, valeurs mobilières [...]). Le pragmatisme du droit commercial au XIXe siècle amène notamment le législateur à faire évoluer le droit. Un dialogue entre les juges consulaires et le législateur se développe ainsi dans le cadre de la première conférence régionale des tribunaux de commerce de la Cour d'appel de Riom.

C'est en particulier le cas, de manière incidente, pour la question de la connaissance des différends qui s'élèveraient à l'occasion d'un contrat de louage entre les patrons et leurs représentants et les employés, ouvriers, apprentis du commerce et de l'industrie. Rapidement évoquée par les membres de la conférence, cette question ne permet pas d'apprécier les arguments des parties. Précisons simplement que les participants sont informés d'un projet de loi (Dutreix) qui soustrairait à la compétence de la juridiction consulaire ce contentieux pour le confier aux Conseils de Prud'hommes ${ }^{13}$. A la lecture du compte rendu de la réunion, on prend

13 Marcel David, L'évolution historique des Conseils de prud'hommes en France, dans: Droit social (1974) 3-21; Françoise Fortunet, D’une République à l'autre. Les Conseils de prud'hommes ou l'institution d'une justice de paix de l'industrie (Justice et république[s], Lille 1993) 325-335; Alain Cottereau, Les prud'hommes au XIX ${ }^{\mathrm{e}}$ siècle. Une expérience originale de pratique du droit, dans: Justices, revue général du droit processuel 8 (1997) 1-8; Jacques Bouveresse, Des élections malgré tout. L'histoire mouvementée des Conseils de prud'hommes (L'élection des juges, étude historique française et contemporaine, Paris 1999) 165-221. 
simplement connaissance de leur refus ${ }^{14}$. Il le justifie par l'absence de création d'une juridiction d'appel spécifique autre que les tribunaux de commerce. C'est là une position qui sera remise en cause par la loi du 15 juillet 1905 qui prévoit que l'appel ne soit plus déféré devant la juridiction commerciale. Par ailleurs la loi du 27 mars 1907 étend la juridiction des prud'hommes aux employés de commerce pour le cas où il serait institué dans cette juridiction une section commerciale (article 1). Elle prévoit aussi que les différends entre patrons et employés ne rentrent pas dans la compétence des «tribunaux ordinaires» (article 32), c'est-à-dire les tribunaux de commerce, au-dessus d'une certaine somme pour la demande formulée $(1000 \text { francs })^{15}$.

La compétence de la juridiction consulaire et la spécificité du contentieux porté devant elle retiennent davantage l'attention des juges des tribunaux de commerce en 1899. Ils alimentent le débat pour améliorer la mise en œuvre de la législation de 1889 relative à la liquidation judiciaire (A) mais aussi pour demander une évolution législative afin de tenir compte des imperfections de la loi de 1898 sur le nantissement $\mathrm{du}$ fonds de commerce (B).

\section{Améliorer la résolution des conflits: l'exemple de la loi du 4 mars 1889 relative à la liquidation judiciaire}

Le difficile équilibre entre les intérêts des créanciers et ceux du débiteur en matière de faillite ne relève pas de l'esprit du Code de commerce de 180716. Ces dispositions se caractérisent par leur sévérité et leur aspect répressif traduisant la volonté politique du régime napoléonien de préservation de l'ordre public. Ces mesures ont alors conduit à une «fuite devant la loi» (Jean Hilaire) par laquelle créanciers et débiteurs ont recherché des solutions plus conformes à leurs intérêts. JeanMarie Pardessus, professeur de droit commercial à Paris au début du XIXe siècle, notait déjà la nécessité d'adopter des mesures différentes pour prendre en considération les comportements coupables ou non des commerçants ${ }^{17}$. Une réforme du

14 BMIUCF, A 11445 , p. 61-62.

15 En ce sens, par exemple, Nîmes, 4 juin 1909, Dalloz (1909), 2, 288.

16 Sur l'évolution du droit des faillites et la bibliographie particulière à cette question, Catherine Labrusse, L'évolution du droit français de la faillite depuis le Code de commerce (Faillites, Paris 1970) 5-54; Marie-Hélène Renaut, La déconfiture du commerçant du débiteur sanctionné au créancier victime, dans: Revue trimestrielle de droit commercial 53-3 (2000) 533-567; Pierre-Cyrille Hautcoeur, Nadine Levratto, Faillite, dans: Dictionnaire historique de l'économie-droit, XVIII $-\mathrm{XX}$ e siècles, sous la direction de Alessandro Stanziani (Droit et société. Recherches et Travaux 17, Paris 2007) 159-167.

17 Jean-Marie Pardessus, Éléments de jurisprudence commerciale (Paris 1811) 507: «Les faillites ont trop d'influence sur la prospérité, le crédit et l'honneur du commerce, pour n'avoir pas dû être l'objet de l'attention spéciale du législateur. Mais il avait, dans cette matière, deux dangers opposés à éviter. S’il importe de mettre les créanciers à même de s'assurer que la faillite de leur débiteur n'est pas l'ouvrage d'une combinaison frauduleuse, ou du moins l'effet d'une imprudence coupable, de punir sans pitié la fraude, d'effrayer l'imprudence par la crainte du déshonneur; il est juste aussi de ne pas enlever au commerçant loyal, que les orages si fréquents dans le commerce ont forcé d'échouer, la consolation de conserver 
droit de la faillite intervient avec la loi du 28 mai 1838 et la loi du 22 juillet 1867 qui abroge la contrainte par corps.

Une nouvelle étape est franchie avec la loi du 4 mars 1889 qui institue la liquidation judiciaire. Elle est prévue pour les commerçants malheureux et de bonne foi. Pour pouvoir bénéficier de la procédure de liquidation judiciaire, le commerçant doit déposer son bilan dans les 15 jours de la cessation de paiement et il ne doit pas exister une cause d'indignité. Le jugement déclaratif n'est alors pas publié. C'est une procédure qui permet le maintien du commerçant-débiteur dans sa gestion avec à ses côtés un liquidateur. Elle permet aussi de conserver au commerçant concerné son honorabilité avec l'exercice maintenu de ses droits électoraux. Si la procédure va à son terme, un concordat est établi avec les créanciers, sinon on revient à la procédure normale de la faillite.

On dispose, par exemple avec les fonds conservés pour le tribunal de commerce d'Aurillac, d'intéressantes données pour apprécier la mise en œuvre de la procédure de la liquidation judiciaire ${ }^{18}$. Les juges sont rapidement confrontés à la nécessité d'être attentifs aux raisons qui justifient la situation du débiteur. De cet examen, le caractère honnête et non frauduleux du commerçant peut être établi ouvrant l'application de la liquidation judiciaire. Dès le mois de juin 1889, les juges consulaires d'Aurillac sont saisis de demandes «suspectes ${ }^{1}{ }^{9}$. Elles illustrent les craintes émises quelques années plus tôt lorsque le projet de loi avait été soumis aux tribunaux pour consultation. Le rapport du président du tribunal de commerce de Thiers en 1885 soulignait alors que la «liquidation judiciaire n'est [...] qu'un leurre ${ }^{20}$. A la lumière de la pratique, la requête adressée au tribunal à partir du délai imparti pour la cessation des paiements pose problème. En effet, bien souvent la date véritable de cessation est antérieure car «ce n'est que complètement acculé», une fois que tout a été tenté que le commerçant se résout à l'inéluc-

l'honneur en perdant la fortune, de lui montrer, dans la sévérité même des épreuves et des vérifications qu'il doit subir, un moyen de mieux manifester son innocence».

18 Archives départementales Cantal, $162 \mathrm{U}$ art 136.

19 Par exemple, un entrepreneur de travaux de l'arrondissement d'Aurillac demande à pouvoir bénéficier de la liquidation judiciaire. Un jugement déclaratif lui en accorde le bénéfice le 29 avril 1889. Mais le juge commissaire de la liquidation présente un rapport tendant à la faire convertir «en faillite, depuis au moins l'année 1884 ». Ayant participé à la construction d'établissements scolaires et réparé l'église de Linvinhac-le-Haut en Aveyron, la gestion de ses affaires est fautive en ce que les travaux ont manqué de surveillance, qu'il a été mis dans plusieurs communes soit en régie soit en demeure de construire des travaux qu'il laissait sans ouvriers et sans matériaux des malfaçons par «sa négligence ou sa force d'inertie». Aussi «ce débiteur [est] harcelé, harassé de demandes en paiements non pas depuis quinze jours comme le ferait supposer sa demande en liquidation mais depuis l'année 1882 et 1883 ». En effet il avait été condamné par la cour de Riom à payer près de 7000 francs en 1884. Le juge commissaire a noté qu'il a obtenu l'année suivante du crédit auprès de différentes banques à Aurillac, à Figeac et à Decazeville. Il demande, à la lumière de l'ensemble de ces éléments, à ce qu'il soit déclaré en état de faillite et que la date de cessation des paiements soit avancée. Il est suivi par le tribunal de commerce qui ordonne le dépôt de l'entrepreneur dans la maison d'arrêt d'Aurillac pour dettes et un jugement du 21 juin fixe l'époque de cessation de paiement au 16 mars 1883.

20 Archives départementales Puy-de-Dôme, 6 BIB 730. 
table. Aussi il en résulte que le tribunal doit faire application de l'article $468 \mathrm{du}$ Code de commerce et le déclarer en faillite. L'espoir de pouvoir jouir des dispositions prévues par la liquidation judiciaire est alors déçu.

L'application de la loi de 1889 ne pouvait manquer de retenir l'attention des juges consulaires au cours de leur première conférence régionale. Faisant le constat que la «bonne foi des juges est trop souvent surprise; [les juges consulaires s'accordent] à reconnaître l'opportunité de couper court à ces abus et de rechercher les moyens d'y parvenir ${ }^{21}$. Les participants à la conférence ne demandent pas une révision de la loi. Ils réfléchissent à la possibilité de mettre en œuvre des mesures qui leur permettent d'être éclairés dans leur décision d'accorder le bénéfice de la liquidation judiciaire. L'enjeu réside dans l'obtention d'informations préalables suffisante pour l'accorder. Ils manifestent ainsi leur «art d'appliquer la loi» (Jean Hilaire). La première mesure proposée tient à l'existence d'une comptabilité régulière du débiteur. La position du tribunal de commerce de Clermont est claire sur ce point. Il déclare ainsi en faillite tout liquidé n'ayant pas de comptabilité c'està-dire l'absence de livres comprenant les registres ordinaires et à jour pour permettre le contrôle de l'exactitude du bilan déposé2 2 . Un deuxième élément d'information mis à la disposition des juges consulaires s'inspire de la pratique d'autres tribunaux de commerce. Comme à Rouen ${ }^{23}$, on décide d'imposer au demandeur lorsqu'il dépose son bilan de répondre à un questionnaire écrit, certifié sincère et signé. La discussion s'engage à partir d'une proposition des juges consulaires de Clermont ${ }^{24}$. Les 17 questions s'attachent à décerner la sincérité de la demande présentée par le commerçant. Il doit préciser s'il est en état de cessation de paiements, s'il y a des protêts, s'il existe des tentatives d'arrangement avec les créanciers, s'il dispose de fonds disponibles ou bien encore quelles sont les raisons de sa situation. Le juge doit pouvoir ainsi connaître la «situation apparente» du commerçant, telle qu'il la déclare, sur le plan financier et commercial mais aussi ses relations avec ses créanciers. Les éléments ainsi renseignés doivent faire l'objet de vérification. Dans un délai de 15 jours, un rapport des syndics est rendu au jugecommissaire de Clermont pour contrôler l'exactitude des réponses données. Deux tendances apparaissent nettement au cours du débat quant à la position des juges après que le dépôt de bilan ait été déposé. La difficulté tient à la position que le juge doit adopter lorsqu'il y a un doute sur la sincérité de la demande du débiteur. Pour les uns, la liquidation judiciaire doit être déclarée immédiatement. On admet de pouvoir déclarer ensuite la faillite à la lumière d'informations plus précises notamment avec la remise du rapport par le syndic dans un délai de 15 jours. La mesure est destinée à préserver l'actif et d'éviter toute action du débiteur au détriment de ses créanciers. C'est la position des tribunaux de Clermont et de Riom. Cette façon de procéder recueille l'assentiment des membres de la conférence. Pour

21 BMIUCF, A 11445, 26.

22 BMIUCF, A 11445, 40.

23 BMIUCF, A 11445, 26.

24 BMIUCF, A 11445, 36. 
d'autres, une enquête rapide doit être faites avant de pouvoir prononcer la liquidation. C'est la pratique observée par le tribunal de commerce de Thiers. La conférence de Rouen avait adopté aussi une telle solution. Cette solution recueille l'assentiment du Président de la Chambre de Commerce de Clermont. Ainsi Chalus, rapporte les propos, allant dans le même sens, de Lyon-Caen pour qui «la garantie du commerçant malheureux, c'est son juge; son danger, c'est son syndic »25. Il insiste alors sur l'importance de l'appréciation de la situation laissée au juge. L'esprit de la loi de 1889 avait cherché à atténuer la rigueur de la situation du débiteur malheureux. Qualifiée de «loi Bérenger du commerce» 26 par un des participants à la Conférence régionale, son application doit rester exceptionnelle pour les juges consulaires ainsi réunis. En cherchant à éclairer leur décision par l'instauration de bonnes pratiques, leur logique poursuit l'objectif de protéger avant tout les créanciers. Le prononcé rapide de la liquidation judiciaire est davantage conçu comme un moyen de préserver l'actif. Cette idée prime sur la volonté affichée de ne pas en faire bénéficier un commerçant malhonnête ${ }^{27}$ car elle peut évoluer vers le prononcé de la faillite. Le même esprit guide les juges consulaires pour l'application de l'article 19 de la loi du 4 mars 1889 qui prévoit la possible transformation de la liquidation en faillite en cas de refus du concordat par les créanciers. Dans une telle hypothèse, les membres de la Conférence affirment que le maintien de la liquidation doit être exceptionnel28.

Les juges des tribunaux de commerce du ressort de la cour d'appel de Riom s'intéressent également à la détermination de la période où a commencé la cessation de paiements qui constitue alors la faillite. Le tribunal doit ainsi fixer (soit par le jugement déclaratif de la faillite, soit par jugement ultérieur, rendu sur le rapport du juge-commissaire) d'office ou sur la poursuite de toute partie intéressée, l'époque à laquelle a eu lieu la cessation de paiements. A défaut, cette cessation est réputée avoir eu lieu à partir du jugement déclaratif de la faillite. Le législateur, par l'article 441 du Code de commerce, leur a laissé un entier pouvoir d'appréciation en ne fixant pas une date limite pour reporter la date de la cessation de paiements dans une faillite. Le débat des représentants des tribunaux de commerce en 1899 tient à la possibilité de fixer une telle limite dans le temps. Leur discussion est éclairée par les positions des conférences d'Orléans (fixation à un an ou au maximum à deux ans le délai en deçà duquel le report d'une faillite peut être prononcé) et de Rouen (refus de fixation d'une limite en raison de fraudes possibles) ${ }^{29}$. L'importance de la liberté d'appréciation des juges consulaires ainsi que la nécessité d'agir en fonction des cas d'espèces les confortent dans leur volonté de ne pas fixer de délai pour le report des faillites. Cette position est présentée comme étant en conformité avec un arrêt de la Cour de cassation du 12 mai $1841^{30}$ précisant que la

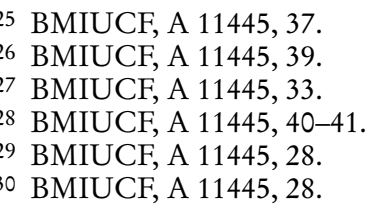


«décision des juges sur l'époque précise de la cessation de paiement étant plus de fait que de droit échappe à la censure de la Cour».

Les juges consulaires réunis à Clermont-Ferrand souhaitent ainsi lutter contre le laxisme d'une application trop générale de la loi de 1889 par l'instauration de pratiques communes. Leur position est tout aussi claire pour attirer l'attention du législateur sur les évolutions à apporter au contentieux en matière de nantissement de fonds de commerce.

\section{Proposer un meilleur règlement des différents: l'exemple de la loi du $1^{\text {er }}$ mars 1898 relative au nantissement $d u$ fonds de commerce}

Le Code de commerce, pas plus que l'ordonnance de 1673, ne se sont intéressés au fonds de commerce, ou de boutique, tant les réticences sont importantes pour consacrer l'existence d'une propriété immatérielle du fonds. La protection du créancier prime sur la possibilité d'accorder du crédit aux boutiquiers sans garantie hypothécaire et en restant dans leur fonds. N'étant pas reconnu comme un patrimoine distinct, il ne peut pas faire l'objet d'un nantissement. Fruit d'une construction du notariat et de la jurisprudence, la reconnaissance légale du fonds, bien que tardive, est apparue nécessaire au XIX $\mathrm{X}^{\mathrm{e}}$ siècle $^{31}$. Cette évolution a été permise dans un premier temps par la loi fiscale du 28 février 1872. Le nantissement du fonds de commerce a suscité à de nombreuses reprises l'intervention du législateur à la fin du XIX ${ }^{\mathrm{e}}$ siècle et au début du XX $\mathrm{XX}^{\mathrm{e}}$ siècle La conférence de Riom de 1899 examine ce sujet pour proposer qu'il soit porté à la connaissance de la conférence générale de Paris.

L'absence de publicité du nantissement du fonds de commerce comme la situation du vendeur du fonds de commerce font naître des incertitudes et des risques dont les juges consulaires souhaitent informer le législateur. Par un arrêt du 13 mars $1888^{32}$, la Chambre des requêtes considère que le fonds de commerce est constitué par l'enseigne, l'achalandage, le droit au bail et les marchandises qui en sont l'accessoire. Ce fonds est susceptible d'être donné en gage et la Cour admet que la dation du nantissement est autorisée à deux conditions qui sont considérées comme nécessaires et suffisantes. D'une part l'acte de nantissement doit être signifié au propriétaire des lieux loués (art. 2075 Code civil). D’autre part le créancier gagiste doit être mis en possession de l'article de bail (art. 2076 du Code civil). La position adoptée par la Cour de Cassation a alors suscité des critiques de la part de la doctrine ${ }^{33}$, en raison du caractère occulte du nantissement, qui permet à un cré-

31 Jean Hilaire, Les contingences historiques du fonds de commerce (L'entreprise personnelle 2, Critique et prospective, Paris 1981) 125-162; repris dans: Jean Hilaire, Le droit, les affaires et l'histoire (Paris 1995) 125-157 (cité comme suit: Hilaire, Le droit); Patrick Le Floch, Le fonds de commerce. Essai sur le caractère artificiel de la notion et ses limites actuelles (Paris 1986).

32 Dalloz (1888) 1, 351.

33 Par exemple, Eugène-Edmond Thaller, Traité élémentaire de droit commercial (Paris ${ }^{41910)}$ 549; cité comme suit: Thaller, Traité. 
ancier unique de disposer en cas de faillite du produit de l'ensemble de l'actif. Des résistances existent aussi de la part de cours d'appel et de tribunaux de commerce. Ils n'admettent pas qu'un commerçant puisse donner en gage le fonds qu'il exploite et ils insistent sur la nécessité d'une publicité en ce que le fonds de commerce constitue le gage commun et apparent des créanciers. Diverses propositions de loi sont débattues en 1893, 1895 et 1897 pour mettre fin à cette résistance grâce à l'action de Millerand. La loi du $1^{\text {er }}$ mars 1898 marque une première évolution en ajoutant un alinéa à l'article 2075 du Code civil précisant que «tout nantissement d'un fonds de commerce devra, à peine de nullité vis-à-vis des tiers, être inscrit sur un registre public tenu au greffe du Tribunal de commerce dans le ressort duquel le fonds est exploité». Thaller juge cependant cette disposition insuffisante. Qualifiée d'hypothèque mobilière, et rapprochée de l'hypothèque existante pour les navires, il regrette ainsi que la loi de 1898 ne prévoit pas un régime de publicité comparable à celui prévue pour les hypothèques immobilières ${ }^{34}$.

Les lacunes législatives n'ont pas manqué de susciter débats ${ }^{35}$ et protestations. Saisie par la Chambre de commerce de Tours, la conférence générale recueille l'avis des juges consulaires sur la loi de $1898^{36}$. Les représentants des tribunaux de commerce du ressort de la Cour d'appel de Riom sont pour le moins réservés d'autant qu'ils sont éclairés par un rapport de la Chambre de commerce de Tours qui envisagent différentes situations commerciales pour démontrer les limites de la loi et même les possibles fraudes qu'elle peut susciter. L'un des risques est notamment constitué par l'entente frauduleuse entre un commerçant, dont la faillite est inéluctable mais qui le sachant accroît ses stocks, et une personne qui devient son créancier muni d'un nantissement. La réalisation de l'actif au profit du créancier gagiste, et la répartition avec le failli, se ferait au détriment des autres créanciers non privilégiés. Jugeant la publicité instaurée par la loi insuffisante pour assurer l'information et la protection des créanciers, les juges consulaires se prononcent pour une modification de la loi. Prenant connaissance par l'intermédiaire du président de la Chambre de commerce de Clermont de l'avis de Lyon-Caen, ils requièrent que des précisions soient apportées sur le mode d'inscription du nantissement, sa durée ou encore la possibilité de radiation. La loi Cordelet du 17 mars 1909, qui consacre l'existence d'une sûreté réelle constituée sur le fonds de commerce sans dépossession du débiteur, apporte les évolutions nécessaires en s'inspirant des règles établies en matière d'hypothèques immobilières sauf à fixer à cinq années la durée d'inscription du nantissement.

En relation avec la question du fonds de commerce, les juges consulaires prennent aussi connaissance des incidences de la loi du $1^{\mathrm{er}}$ mars 1898 en matière de faillite. La difficulté débattue par les juges consulaires et faisant l'objet d'un contentieux est celle de la situation du vendeur d'un fonds de commerce. Deux hypothèses doivent être distinguées selon que l'acheteur tombe ou non en faillite. Lorsque

34 Thaller, Traité (n. 33) 550.

35 Bulletin de la Société d'Etudes législatives (1903), 218.

36 BMIUCF, A 11445, 64. 
celui-ci ne connaît pas de difficultés, le vendeur dispose d'un privilège et d'un droit de suite pour lui garantir le paiement du prix par l'acheteur ou par un sousacquéreur. Considéré comme un meuble, le fonds de commerce voit alors s'appliquer le droit général de la vente mobilière. A défaut de paiement, le vendeur est ainsi autorisé à poursuivre la résolution (articles 1184 et 1654 du Code civil). Une seconde hypothèse correspond à la situation de faillite dans laquelle se trouve l'acheteur. La reconnaissance de la double garantie réelle au profit du vendeur du fonds en fait un créancier placé dans une meilleure situation que celle de toute autre créancier vendeur de meubles, en particulier les fournisseurs. Un problème existait alors dans la vie du commerce au début du XIXe siècle en cas de faillite relativement à la différence de situations des créanciers, certains bénéficiant d'un privilège ou d'un droit de préférence et d'autres non ${ }^{37}$. Cette situation est d'autant plus délicate qu'il n'y avait pas de publicité des ventes de fonds de commerce avant que la pratique puis le législateur l'organisent ${ }^{38}$. La volonté du législateur a alors été d'établir une égalité entre les créanciers du commerçant failli avec la loi de 1838. Ainsi par l'article $550 \mathrm{du}$ Code de commerce, il est prévu en son dernier paragraphe, que "privilège et droit de revendication établis par le $\mathrm{n}^{\circ} 4$ de l'article 2102 du Code civil au profit du vendeur d'effets mobiliers, ne seront point admis en cas de faillite». Complété par l'article 576 du Code de commerce, le privilège et l'action résolutoire du vendeur sont ainsi supprimés en cas de faillite de l'acheteur $\mathrm{du}$ fonds de commerce. Pour améliorer leur situation, les vendeurs ont eu recours à la loi du $1^{\text {er }}$ mars 1898 en stipulant un nantissement sur le fonds de commerce qu'ils aliénaient. On y vit un moyen de contourner les articles 550 et 576 du Code de commerce. Aussi la loi de 1898 aboutit alors pour certains à «virtuellement abroger» $\operatorname{ces}_{\text {articles }}{ }^{39}$. La loi du 17 mars 1909 décide alors que l'article 550 du Code de commerce ne serait applicable ni au privilège ni à l'action résolutoire du vendeur d'un fonds de commerce. Elle a rendu possible de rendre public le privilège $\mathrm{du}$ vendeur de fonds de commerce par son inscription sur un registre du greffe du tribunal de commerce dans le ressort duquel le fonds est exploité.

L'examen de difficultés résultant de l'application de la loi par les juges consulaires motive la première conférence régionale des tribunaux de commerce du ressort de la cour d'appel de Riom à prendre part au débat pour des domaines qui relèvent de leur compétence. Les représentants des tribunaux de commerce sont aussi attentifs aux différences d'appréciation voire aux concurrences de compétences entre les juridictions commerciales et civiles. La spécificité et l'efficacité du contentieux commercial sont alors présentées comme des revendications d'une identité qu'ils considèrent menacée.

37 Dalloz (1884) 1, 241. Dalloz (1891) 1, 415.

38 Thaller, Traité (n. 33) 67-68.

39 BMIUCF, A 11445, 65. 


\section{Les juges consulaires et le juge civil}

Thaller précisait que si les «tribunaux de commerce son absolument incompétents en matière civile. Il apparaitrait logique de décider que tribunaux civils sont, de leur côté, absolument incompétents en matière commerciale. Or cette réciprocité n'est pas acceptée [...] Les tribunaux civils, étant tribunaux de droit commun, disposent de la plénitude de juridiction, et possèdent un germe de compétence [...] même sur les matières de commerce ${ }^{40}$. L'évocation des rapports ente ces deux juridictions au cours de la conférence régionale suscite un «réflexe identitaire» de la part des représentants des tribunaux de commerce. Ils cherchent à affirmer d'une part la particularité et les avantages «classiques» de la procédure en matière commerciale (A) et ils souhaitent d'autre part que cette juridiction d'exception connaisse l'application de règles de procédure observées par les tribunaux civils pour ne plus être «subordonné» à eux (B).

\section{Défendre l'efficacité de la résolution des conflits en matière commerciale}

La procédure commerciale est guidée depuis le Moyen Âge par le souci de la rapidité, de la simplicité et de l'efficacité. Les règles de procédure devant les tribunaux de commerce ont été précisées dans le titre XXV du livre II de la première partie du Code de procédure civile (articles 414 à 442). Les juges consulaires prennent position pour défendre cette spécificité en examinant de manière générale le recours à la conciliation en matière commerciale et en demandant de manière plus particulière l'abrogation de l'article $427 \mathrm{du}$ Code de procédure civile relatif au renvoi par la juridiction consulaire devant la justice civile de l'affaire dans laquelle une pièce produite est méconnue, déniée ou arguée de faux.

En raison de la longueur et du coût des procès, la conciliation est souvent envisagée comme une voie à suivre ${ }^{41}$. La conciliation a été mise en place par l'Assemblée constituante avec la loi du 20 août 1790 créant les juges de paix. Le recours systématique à la conciliation, avant l'instance, devait susciter des difficultés et des critiques car elle allongeait parfois inutilement la résolution d'une affaire. Il y avait là une limite pour la résolution des différents commerciaux. Le législateur napoléonien a précisé les modalités du recours à la conciliation en apportant des restric-

40 Thaller, Traité (n. 33) 1103-1104.

41 Joseph-Edouard Boitard, Leçons de procédure civile t. 1 (Paris, 151890) 73 (cité comme suit: Boitard, Leçons), évoque Voltaire pour qui «la meilleure loi, le plus excellent usage, le plus utile que j'aie jamais vu, c'est en Hollande. Quand deux hommes veulent plaider l'un contre l'autre, ils sont obligés d'aller d'abord au tribunal des conciliateurs, appelés faiseurs de paix. Si les parties arrivent avec un avocat ou un procureur, on fait d'abord retirer ces derniers, comme on ôte le bois d'un feu qu'on veut éteindre. Les faiseurs de paix disent aux parties: Vous êtes de grands fous de vouloir manger votre argent à vous rendre mutuellement malheureux: nous allons vous accommoder sans qu'il vous en coûte rien». 
tions avec les articles $48^{42}$ et 4943 du Code de procédure civile. Ce dernier exclue les affaires commerciales par deux dispositions qui ont été perçues comme redondantes en visant «les demandes qui requièrent célérité» $\left(2^{\circ}\right)$ et «les demandes en matière de commerce» $(4 \circ)$. Est-ce à dire que la conciliation n'existait pas en matière commerciale? Évidemment non. Le débat des juges consulaires, réunis à la conférence régionale, sur la place de la conciliation dans les affaires du commerce met en lumière les pratiques observées et la volonté d'affirmer quelques principes.

Outre la question de la répartition des frais liés à la conciliation pris en charge par les parties, une position générale est adoptée décidant de «rejeter l'obligation de la tentative préliminaire pour éviter tout retard dans l'introduction de l'instance et par suite dans le jugement ${ }^{44}$. Le refus est exprimé que la conciliation intervienne avant assignation contrairement à la pratique observée à Tours ${ }^{45}$. Les juges consulaires souhaitent que les tribunaux jouissent de la faculté de mettre en œuvre les moyens qu'ils jugent appropriés et de laisser aux parties la liberté d'y avoirs recours. Il leur importe qu'un juge intervienne à un moment ou à un autre de la tentative de conciliation. Loin de s'opposer au principe même, ils envisagent de proposer différents moyens avec le renvoi devant un juge conciliateur, la comparution des parties en chambre du Conseil ou bien encore la nomination d'arbitres commerçants $^{46}$. A la lumière de l'expérience, la première solution leur apparaît plus adaptée pour des affaires peu importantes. Dérogeant au principe général qu'ils formulent, la proposition est néanmoins faites pour ce contentieux de rendre obligatoire et préalable la conciliation. Eclairé par Vindry, l'ancien président du tribunal de commerce de Lyon, les membres de la conférence régionale sont informés des modalités de la conciliation observée dans sa juridiction ${ }^{47}$. Telle une profession de foi, il déclare que lorsque «nous avons l'assignation [...] nous devons faire tout ce que nous pouvons pour tâcher de les faire transiger et s'ils ne transi-

42 Article du 48 Code de Procédure Civile, «Aucune demande principale introductive d'instance, entre parties capables de transiger, et sur des objets qui peuvent être la matière d'une transaction, ne sera reçue dans les tribunaux de première instance, que le défendeur n'ait été préalablement appelé en conciliation devant le juge de paix, ou que les parties n'y aient volontairement comparu».

43 Article 49 du Code de Procédure Civile, «Sont dispensés du préliminaire de la conciliation: $1^{\circ}$ les demandes qui intéressent l'Etat et le domaine, les communes, les établissements publics, les mineurs, les interdits, les curateurs aux successions vacantes; $-2^{\circ}$ les demandes qui requièrent célérité; $-3^{\circ}$ les demandes en intervention ou en garantie; $4^{\circ}-$ les demandes en matière de commerce; - $5^{\circ}$ les demandes de mise en liberté, celles en mainlevée de saisie ou opposition, en paiement de loyers, fermages, arrérages de rentes ou pensions; celles des avoués en payement de frais; $-6^{\circ}$ les demandes formées contre plus de deux parties, encore qu'elles aient le même intérêt; - 7o les demandes en vérification d'écriture, en désaveu, en règlement de juges, en renvoi, en prise à partie; les demandes contre un tiers saisi, sur les offres réelles, sur la remise des titres, sur leur communication, sur les séparations de biens, sur les tutelles et curatelles; et enfin toutes les causes exceptées par les lois».

44 BMIUCF, A 11445, 32.

45 BMIUCF, A 11445, 31-32.

46 BMIUCF, A 11445, 51-55.

47 BMIUCF, A 11445, 53. 
gent pas, nous jugeons» ou encore que «nous faisons de la conciliation tant que nous pouvons ${ }^{48}$. Elle s'appuie sur l'organisation en sections, selon la nature des affaires, du Tribunal de commerce. La pratique suivie à Lyon repose alors, en relation avec le rôle des appels de causes, sur l'intervention d'un juge possédant de l'expérience («subtil, rapide, sûr de lui») pour renvoyer certaines affaires devant les sections pour conciliation. Les parties sont ainsi entendues et les juges proposent soit le prononcé d'un jugement soit une tentative de conciliation devant la chambre du conseil (article 428 du Code de procédure civile). Pour certaines affaires particulières, notamment celles relatives aux chemins de fer, le tribunal de commerce de Lyon renvoie toujours en conciliation devant deux ou trois sections. Enfin, parmi les possibilités de concilier les parties, les juges consulaires peuvent désigner un arbitre. Les articles 428 et 429 du Code de procédure civile permettent notamment l'intervention d'arbitres conciliateurs ou à défaut de rapporteurs ${ }^{49}$.

La conciliation a été ainsi organisée de manière pratique par les tribunaux de commerce. Thaller rapporte que les transactions en matière commerciales sont deux fois plus nombreuses qu'en matière civile représentant ainsi près de $30 \%$ des modes de règlements de différends qu'il explique comme la manifestation de «ménager un adversaire pour ne pas rompre dans l'avenir le lien de la clientèle ${ }^{50}$. S'il a réussi à faire accepter l'idée qu'il ne devait pas y avoir de tentative obligatoire de conciliation pour les procès commerciaux, le juge consulaire ne s'interdit pas d'essayer de concilier les parties. Pour l'article 427 du Code de procédure civile ${ }^{51}$, les juges consulaires sont également soucieux de la rapidité de la procédure ${ }^{52}$. Cet article leur apparaît être un obstacle. Dans les cas visés, le tribunal de commerce doit surseoir à statuer sur la demande principale pour renvoyer l'affaire devant le tribunal civil. En de nombreux cas, notamment pour le paiement d'une lettre de change ou d'un billet à ordre, le tribunal de première instance est amené à être saisi de l'incident mais non du fonds. La critique formulée est alors triple. La première tient à la pratique de nomination d'un expert par la juridiction de droit commun qui ralentit d'autant la marche du procès. Un jugement du tribunal de commerce doit renvoyer la vérification d'écriture devant le tribunal civil. Celui-ci va nommer un expert puis rendre un jugement sur l'expertise. La seconde critique a trait à la portée relative et contestable des conclusions des experts. Ainsi l'article 427 est perçu comme «une entrave, sans compensation pour le justiciable, apportée à l'exercice de la justice consulaire » ${ }^{53}$. Enfin, les membres de la conférence réfutent la compétence de la juridiction civile qui serait justifiée par la présence d'un repré-

48 BMIUCF, A 11445, 53-54.

49 Article du 429 Code de Procédure Civile, «S'il y a lieu à renvoyer les parties devant les arbitres pour examiner des comptes, pièces et registres, il sera nommé un ou trois arbitres pour entendre les parties et les concilier si faire se peut, sinon donner leur avis [...]».

50 Thaller, De l'avenir (n. 11) 209.

51 Article 427 du Code de Procédure Civile, «Si une pièce produite est méconnue, déniée ou arguée de faux et que la partie persiste à s'en servir, le Tribunal (de commerce) renverra devant les juges qui doivent en connaitre; il sera sursis au jugement de la demande principale».

52 BMIUCF, A 11445, 58.

53 BMIUCF, A 11445, 59. 
sentant du ministère public pouvant intervenir en cas de présomption de faux. L'importance de cette présence a évolué au cours du XIX ${ }^{\mathrm{e}}$ siècle pour les tribunaux civils jugeant commercialement. Par un arrêt de la cour de Rennes du 23 décembre 1816, il était admis l'absence du ministère public. Une jurisprudence contraire a été établie par la Cour de cassation qui a exigé sa présence à l'audience commerciale des tribunaux civils (arrêt 21 avril 1846 et 15 juillet 1846) ${ }^{54}$. Les juges consulaires demandent une évolution législative pour leur permettre de saisir le Procureur de la République, absent de leur audience. N'ayant plus à surseoir à statuer, ils pourraient aussi contribuer à la défense de la société. Il ne serait alors pas besoin que la juridiction civile connaisse de l'incident en raison de la présence d'un représentant du ministère public. Il faut attendre la loi du 10 juillet 1970 pour que le Procureur de la République soit autorisé à exercer ses attributions devant les juridictions d'exception.

Les juges des tribunaux de commerce formulent clairement la proposition de la suppression de cet article pour réserver à eux seuls la connaissance de cette question. A l'appui de cette demande, ils évoquent deux raisons principales. Tout d'abord, on a pu faire remarquer que la Cour de cassation admet notamment par des arrêts du 4 août 188455 , du 1er juin $1892^{56}$ et du 26 juin 189457 que les juges devant lesquels une écriture ou signature est déniée peuvent procéder eux-mêmes à la vérification, sans avoir recours à une enquête ou expertise, à l'aide des pièces, documents ou circonstances de la cause. Le président du tribunal de commerce de Saint-Étienne rapporte diverses décisions en faveur des juridictions consulaires. Ainsi un arrêt de la Cour d'appel d'Aix du 22 février 1870 a jugé que le tribunal de commerce, devant lequel une partie actionnée en exécution d'un engagement dénie sa signature, n'est pas tenu de surseoir pour la vérification préalable de la pièce par la juridiction civile ${ }^{58}$. Cette possibilité lui est reconnue si l'exception lui paraît n'être pas sérieuse mais imaginée seulement pour les besoins de la cause. Divers jugements consulaires dans les années 1895-1897 adoptent une position semblable ${ }^{59}$. Toutefois, la jurisprudence à la fin du XIX ${ }^{\mathrm{e}}$ siècle ne reconnait pas cette faculté aux juridictions consulaires ${ }^{60}$. Les représentants des tribunaux de commerce du ressort de la Cour d'appel de Riom rappellent qu'elle adopte une jurisprudence opposée. Une seconde raison à l'appui de la demande d'abrogation de l'article 427 du Code de procédure civile tient à l'expérience particulière des juges du commerce pour apprécier ces situations ${ }^{61}$ : «Les Tribunaux de commerce ont été institués afin que les difficultés qui surviennent à la suite des opérations commerciales

54 Dalloz (1846), 1, 131 et 270. Voir aussi Cour de cassation, 12 juill. et 24 nov. 1847, Dalloz (1847), 1, 255 et (1847), 4, 484; Cour de cassation 5 avril 1848, Dalloz (1848), 5, 361.

55 Dalloz (1885) 1, 209.

56 La loi (1892) 1087.

57 Gazette du Palais (1894) 1, 228.

58 Dalloz (1870) 2, 190.

59 BMIUCF, A 11445, 60.

60 Paris, 5 avril 1884, Sirey (1884) 2, 166.

61 BMIUCF, A 11445, 58. 
soient tranchées par ce qu'on appelle des professionnels, c'est-à-dire par des hommes habitués aux affaires et devenus, par la pratique, compétents pour apprécier les faits et résoudre rapidement et autant que possible par eux-mêmes et sans le concours d'experts les procès portés devant eux». Leur légitimité justifie alors pour eux qu'ils connaissent du principal et des incidents. Ils affirment ici, comme à l'égard des experts ou des conseils de prud'hommes, leur compétence. On peut y voir à propos de cette question, mais de manière plus générale avec la conférence régionale, une manière de réaffirmer leur utilité, leur spécificité et sans doute dans une certaine mesure leur identité comme juridiction d'exception pour résoudre les conflits en matière de commerce. Cette identité propre transparaît également par le mode de désignation des magistrats. Juridiction d'exception, le tribunal de commerce se caractérise par la présence de magistrats élus et temporaires. Les juges consulaires réunis en 1899 à Clermont-Ferrand proposent de porter à la connaissance de la Conférence générale une demande de modification de la loi du 8 décembre 1883 relative aux élections consulaires (élection des magistrats au scrutin de liste avec désignation sur une liste unique des fonctions dévolues à chaque candidat ${ }^{62}$.

Malgré leur composition, leur compétence et leur procédure différente, les tribunaux de commerce et les tribunaux civils entretiennent des «liens» hérités du passé que les juges consulaires du ressort de la cour d'appel de Riom prennent en considération.

\section{Rapprocher juridiction d'exception et de droit commun}

Au cours de la première conférence régionale, les représentants des tribunaux de commerce manifestent la volonté que les règles applicables devant les juridictions civiles puissent s'appliquer en matière commerciale dans un souci de plus grande efficacité, en particulier pour l'exécution des jugements. En d'autres domaines, ils manifestent le souhait d'un maintien des juridictions d'exception au seul premier degré en s'opposant à la proposition de création de tribunaux d'appel spécifiques pour la matière commerciale.

La conférence des tribunaux de commerce du ressort de la cour d'appel de Riom souhaite que la conférence générale de Paris examine deux questions relatives à la procédure. Elle défend l'idée d'un même traitement réservé à la juridiction d'exception qu'aux juridictions de droit commun. La première demande porte sur l'article 442 du Code de procédure civile par lequel «les tribunaux de commerce ne connaîtront point de l'exécution de leurs jugements». Cette disposition est justifiée généralement par l'idée que la question de l'exécution du jugement définitif d'un tribunal de commerce n'est pas une question commerciale ${ }^{63}$. Les jugements préparatoires et interlocutoires ne sont pas considérés comme étant visés par l'article 442 (sous réserve de l'article 427 que nous avons déjà rencontré). On distin-

62 BMIUCF, A 11445, 55-57.

63 En ce sens, par exemple, Boitard, Leçons, t. 2 (n. 41) 40. 
gue aussi entre ce qui relève de l'exécution proprement dite et les difficultés liées à l'explication, l'interprétation ou encore l'application d'un des chefs contenus dans le jugement. La Cour de cassation par un arrêt du 4 mai 1869 s'est ainsi prononcée pour qu'un tribunal de commerce connaisse de difficultés liées au caractère des actes d'exécution ${ }^{64}$. La seconde demande est relative à la procédure de saisie et à l'application des articles 563 et suivants du Code de procédure civile65. Leur volonté est que la demande puisse être introduite devant la juridiction commerciale. Dans un différent commercial, les juges consulaires attirent l'attention sur l'obligation pour le créancier saisissant d'assigner en validité devant le tribunal civil le débiteur saisi. Les tribunaux de commerce ne peuvent ainsi pas connaître de l'exécution de leurs jugements en ce qui concerne la saisie de la personne du débiteur ou de ses biens. Mais ils ont le droit de prononcer la contrainte par corps contre toute personne, même non commerçante, qui est condamnée pour dette commerciale au paiement d'une certaine somme (fixée à au moins 200 francs par la loi du 17 avril 1832). L'existence d'exceptions à son application et sa mise en œuvre limitent d'autant sa portée en matière commerciale.

Les membres de la conférence sont enfin saisis de la proposition de la création d'une juridiction spéciale d'appel pour connaître des jugements des tribunaux de commerce. La proposition revient à Félix Faure de constituer des cours d'appel commerciales dont les membres seraient nommés par les tribunaux et les chambres de commerce ${ }^{66}$. La lecture du compte rendu des travaux de la conférence auvergnate permet de saisir les arguments avancés dans le débat. Pour certains, en faveur d'une telle création, les juges de première instance comme ceux d'appel doivent présenter les mêmes caractéristiques. On doit ainsi avoir dans les deux degrés de juridiction des «juges de faits et professionnels». En outre, l'extension des qualités de simplicité et de rapidité de la procédure commerciale au deuxième degré apparaîtrait bénéfique ${ }^{67}$. La position de la conférence de Clermont rejoint celle tenue à Orléans pour refuser une telle innovation en raison des difficultés de recrutement de ces juges du second degré mais aussi de certaines limites liées à leur formation. On affirme que les «juges d'appel, pris forcément par les anciens présidents et juges consulaires, formés par conséquent dans le même esprit, seraient vraisemblablement exposés, en raison de leurs connaissances juridiques imparfaites, [...], à prononcer [...] avec sentiment plutôt qu'avec application stricte de la doctrine ${ }^{68}$. Les magistrats de carrière offrent ainsi une garantie que les sentences

64 Dalloz (1869) 1, 518.

65 En particulier, article 563 du Code de Procédure civile: «Dans la huitaine de la saisie-arrêt ou opposition [...] le saisissant sera tenu de dénonce la saisie-arrêt ou opposition au débiteur saisi et de l'assigner en validité» et article 567 du Code de Procédure Civile: «La demande en validité, et la demande en mainlevée formée par la partie seront portées devant le tribunal du domicile de la partie saisie».

66 Thaller, Traité (n. 33) 1107 et Annales de droit commercial (1886) 120, «On en voit pas [...] que la magistrature du second degré ait démérité de la confiance que les plaideurs du commerce comme les autres lui ont toujours témoignée».

67 BMIUCF, A 11445, 28.

68 BMIUCF, A 11445, 29. 
des tribunaux soient «conformes non seulement aux grands principes, mais aussi au texte même du droit commercial» 69 . Un statu quo est ainsi défendu avec le maintien de l'appel des jugements des tribunaux de commerce devant les cours d'appel.

Au XIXe siècle, le débat législatif a été alimenté par différentes institutions qui avaient une connaissance de la pratique des affaires. Outre, par exemple, les Chambres de commerce ${ }^{70}$, l'Union des banquiers des départements ou encore la Société d'Etudes législatives, la place des tribunaux de commerce mérite d'être aussi mise en lumière. Les conférences régionales des tribunaux de commerce poursuivent un objectif commun de représentation, de réflexion et de proposition liés aux intérêts du commerce auprès des pouvoirs publics pour les questions liées notamment à la résolution des conflits. De la réunion clermontoise, l'importance d'un règlement des différends rapide et efficace est clairement réaffirmée. C’est le sens de leurs inquiétudes ou prises de position relatives à la liquidation judiciaire, au nantissement ou à l'article $427 \mathrm{du}$ Code de procédure civile. Ils rappellent aussi que cette résolution des conflits du commerce doit relever de professionnels issus du milieu des affaires. La connaissance de la pratique et des usages est indispensable au premier degré. La juridiction consulaire est une juridiction d'exception et elle entend le rester. Il conviendrait de mieux connaître le rôle de ces conférences régionales et de leurs relations avec la Conférence générale des Tribunaux de commerce de France instituée à la fin du XIXe siècle, et devenue depuis 2007, la Conférence des Juges Consulaires, poursuivant des objectifs communs tels le développement de relations «cordiales et solidaires» entre ses membres mais aussi de représentation et de réflexion auprès des pouvoirs publics pour réfléchir notamment aux modalités de résolution des conflits en matière commerciale.

\section{Kurzfassung}

Die Geschichte der Konsulargerichtsbarkeit in der Auvergne ist weitgehend unerforscht. Indes erhellt der Compte rendu de la Conférence régionale des juges consulaires du ressort de la cour d'appel de Riom en 1899 für die erste Regionalkonferenz der gewählten Handelsrichter im Zuständigkeitsbereich des Berufungsgerichts von Riom die unterschiedlichen Problemfelder, auf denen sich die Konsulargerichtsbarkeit im Rahmen handelsrechtlicher Konfliktlösung bewegt: Handelsstreitigkeiten, Kompetenzkonflikte mit der Zivilgerichtsbarkeit, Konflikte der, in einem weiteren Sinne, „privaten Rechtspflege“, die sich des Vergleichs bedient.

Hinsichtlich des Verfahrensrechts und des materiellen Rechts von großem Interesse, zeigt die bewusste Versammlung die Wahrnehmung, welche die Konsular-

69 BMIUCF, A 11445, 29.

70 Hilaire, Le droit (n. 31) 116-119. 
richter von ihrer eigenen Gerichtsbarkeit haben. Die Überlegungen der Richter offenbaren ein doppeltes Interesse: Sie gehen einerseits über den engeren Bezugsrahmen der Konsulargerichtsbarkeit im Zuständigkeitsbereich des Berufungsgerichts von Riom und über deren Schwierigkeit, die Lage anderer Sondergerichtsbarkeiten zu erfassen, hinaus. Andererseits gewährt die bewusste Versammlung, in Verbindung mit der Konferenz der Handelsgerichtspräsidenten, Einblick in Stellung und Bedeutung der Konsulargerichtsbarkeit innerhalb der Fortentwicklung der Handelsgerichtsbarkeit am Ende des 19. Jahrhunderts, in Sonderheit, dafür, die Abgrenzung ihrer Kompetenz gegenüber den gemeinrechtlichen Gerichtsbarkeiten wieder und wieder zu bekräftigen oder weiterzuentwickeln.

Dieser Beitrag zielt somit darauf, die Bedeutung einer dieser Regionalkonferenzen und ihrer Verbindungen mit der Generalkonferenz der französischen Handelsgerichte klarer hervortreten zu lassen. Eingerichtet wurde diese am Ende des 19. Jahrhunderts und im Jahr 2007 wurde sie zur Konferenz der Konsularrichter, welche gemeinsame Ziele verfolgt, wie etwa die Entwicklung von „einträchtigen und solidarischen" Beziehungen zwischen ihren Mitgliedern, aber auch die Interessenvertretung gegenüber den Behörden, mit dem Ziel, in Sonderheit die Modalitäten handelsrechtlicher Konfliktlösung abzuwägen. Infolge ihres Praxisbezugs bekräftigen die Konsularrichter ihre Absicht, das gesetzgeberische Handeln transparent werden zu lassen („Les juges consulaires et le législateur“). Der besondere Charakter von Handelsstreitigkeiten und ihrer rechtlichen Behandlung bringt die Konsularrichter ferner dazu, sich selbst in Bezug auf die Zivilrichter näher zu bestimmen („Les juges consulaires et le juge civil“).

\section{Abstract}

The history of the consular justice of the Auvergne is not well known. But the report of the first regional conference of the consular judges at the court of appeal at Riom in 1899 sheds a light on the different types of problems in connection with the resolution of conflicts. It was question of commercial disputes, competence conflicts with the civil courts or with "private justice" in a larger sense, i.e. including disputes which were solved by settlement or other transactions. Interesting from a procedural and fundamental point of view, this reunion focused also on the self-perception the consular judges had of their own jurisdiction. Their reflections present a double interest. For one part, they reflect on the narrow confinement of the consular jurisdictions within the district of the court of appeal of Riom and their problems with considering the situation of other institutions of exception. Secondly, this reunion, in relation with the conference of the presidents of the commercial courts, permits to understand the place and the role of the consular jurisdictions in the evolution of the commercial law at the end of the $19^{\text {th }} \mathrm{c}$. , especially to affirm, reaffirm or enlarge the limits of their competence in proportion to the general courts of common law. This article therefore proposes to better 
understand the role of one of the regional conferences and its relation with the general conference of the commercial courts of France which was founded at the end of the $19^{\text {th }} \mathrm{c}$. and in 2007 became the "Conférence des Juges Consulaires". It pursues common aims like the development of cordial and solidary relations between its members and their representation but also reflection of the public authorities on the modalities in which to resolve commercial conflicts. Based on their practical knowledge, the consular judges thus confirm their will to clarify the activities of the legislator («Les juges consulaires et le législateur»). The particularities of the commercial trials and their procedure also lead them to determine their role in relation to the civil judge («Les juges consulaires et le juge civil»). 